\section{(1)}

CrossMark

\title{
In-hospital mortality following lung cancer resection: nationwide administrative database
}

\author{
Pierre-Benoit Pagès ${ }^{1,2}$, Jonathan Cottenet ${ }^{3}$, Anne-Sophie Mariet $^{3}$, \\ Alain Bernard ${ }^{1}$ and Catherine Quantin ${ }^{3,4,5}$
}

Affiliations: ${ }^{1}$ Dept of Thoracic Surgery, Centre Hospitalier Universitaire Dijon, Bocage Central, Dijon, France. ${ }^{2}$ INSERM UMR 866, Centre Hospitalier Universitaire Bocage, University of Burgundy, Dijon, France. ${ }^{3}$ Department of Biostatistics and Medical Informatics, Centre Hospitalier Universitaire Dijon, Bocage Central, Dijon, France. ${ }^{4}$ INSERM, CIC 1432, Clinical Investigation Centre, Clinical Epidemiology/Clinical Trials Unit, Centre Hospitalier Universitaire Bocage, University of Burgundy, Dijon, France. ${ }^{5}$ INSERM UMR 1181, Biostatistics, Biomathematics, Pharmacoepidemiology and Infectious Diseases, Centre Hospitalier Universitaire Bocage, University of Burgundy, Dijon, France.

Correspondence: Pierre-Benoit Pagès, Department of Thoracic Surgery, CHU Dijon Bocage Hospital, 14 rue Gaffarel, BP 7790821079 Dijon, France. E-mail: pierrebenoit.pagesQchu-dijon.fr

ABSTRACT Our aim was to determine the effect of a national strategy for quality improvement in cancer management (the "Plan Cancer") according to time period and to assess the influence of type and volume of hospital activity on in-hospital mortality (IHM) within a large national cohort of patients operated on for lung cancer.

From January 2005 to December 2013, 76235 patients were included in the French Administrative Database. Patient characteristics, hospital volume of activity and hospital type were analysed over three periods: 2005-2007, 2008-2010 and 2011-2013.

Global crude IHM was 3.9\%: 4.3\% during 2005-2007, 4\% during 2008-2010 and 3.5\% during 2011-2013 $(\mathrm{p}<0.01) .296,259$ and 209 centres performed pulmonary resections in 2005-2007, 2008-2010 and 2011-2013, respectively $(\mathrm{p}<0.01)$. The risk of death was higher in centres performing $<13$ resections per year than in centres performing $>43$ resections per year (adjusted (a)OR 1.48, 95\% CI 1.197-1.834). The risk of death was lower in the period 2011-2013 than in the period 2008-2010 (aOR 0.841, 95\% CI 0.764-0.926). Adjustment variables (age, sex, Charlson score and type of resection) were significantly linked to IHM, whereas the type of hospital was not.

The French national strategy for quality improvement seems to have induced a significant decrease in IHM.

@ERSpublications

In France, in-hospital mortality following lung cancer surgery is significantly linked to hospital volume http://ow.ly/YgbAy 


\section{Introduction}

Currently, pulmonary resection remains the most efficient treatment for lung cancer [1]. However, despite improvements in the quality of care for lung cancer, pulmonary resection is still associated with postoperative mortality ranging from $2.5 \%$ to $3 \%$, according to large databases $[2,3]$. The quality of care depends on structure, process and outcomes $[4,5]$. Structure relates to the organisation of care, such as the number of beds in hospitals or the number of nurses; process relates to actual actions of care, such as whether the patient receives medication or surgery within a certain timeframe; and outcomes include patient outcome measures such as in-hospital mortality (IHM). Differences in IHM between centres could be explained not only by the quality of care, but also by patient characteristics, registration bias and residual confounding $[5,6]$.

In 2009, a national strategy for quality improvement in cancer management (the "Plan Cancer"), which included new measures, was implemented to improve cancer care and was imposed in French hospitals [7]. These measures concerned cancer research, screening and the medical and surgical management of lung cancer patients. To obtain authorisation, the surgical team has to achieve $>30$ lung resections per year [8].

Few studies have used national databases to analyse the evolution of practices for lung cancer surgery over a defined period [2,9-14], and few studies based on reports from institutions or the literature have been published [15-19]. It remains difficult to interpret the results of these studies because of the lack of data uniformity and the heterogeneity between single and/or specialised centres participating in these studies. This is why a national administrative database is an important tool to assess the quality of care, as it takes into account the characteristics of patients and all centres in the country. The French national administrative database for hospital care (Programme de Médicalisation des Systèmes d'Information (PMSI)) provides a huge amount of epidemiological information concerning hospitalised French patients [20-22]. Data pertaining to pulmonary resection for lung cancer are reliable enough to count such patients.

This nationwide survey was set up to monitor the performance of institutions using a framework of structure and outcome indicators, such as IHM. 5 years after the implementation of the national strategy for quality improvement Plan Cancer, we were interested to know the impact of this new organisation and the establishment of the threshold for hospital volume of activity for lung cancer surgery.

The objective of the present study was to estimate the period effect and to assess the influence of hospital volume on IHM within a large national cohort of patients operated on for lung cancer.

\section{Materials and methods}

\section{Data source and study population}

All data for patients who underwent pulmonary resection for lung cancer in France were collected from January 2005 to December 2013 from the national administrative database. This database was inspired by the US Medicare system. The reliability and validity of PMSI data have already been assessed [23]. Routinely collected medical information includes the principal diagnosis, secondary diagnoses and procedures performed. Diagnoses identified during the hospital stay are coded according to the International Classification of Diseases, 10th revision (ICD-10) [24]. We selected patients in whom a diagnosis of primary lung cancer was coded as the principal discharge diagnosis (all codes C34). Procedures are coded according to the common classification of medical procedures (Classification Commune des Actes Médicaux). For all patients, lung cancer was proven by pathology analyses according to the 2004 World Health Organization classification of lung cancer [25]. Surgery-related variables included the surgical approach (thoracotomy or video-assisted thoracic surgery), the type of resection (limited resection, lobectomy, bilobectomy and pneumonectomy), bronchoplasty and the extent of the pulmonary resection (to the chest wall, the left atrium, the carina, the diaphragm and the superior vena cava).

\section{Patient characteristics}

Baseline demographics included age and sex. From the national administrative database, we included the following comorbidities: pulmonary disease (chronic bronchitis of emphysema), heart disease (coronary artery disease, cardiac arrhythmia, congestive heart failure, valvular heart disease, pulmonary artery hypertension or pulmonary embolism), peripheral vascular disease, alcoholism, liver disease, cerebrovascular events, neurological diseases (hemiplegia or paraplegia), dementia, diabetes mellitus without complications, diabetes mellitus with complications, renal disease, coagulopathy, leukaemia, lymphoma, ulcer disease, history of malignant disease, obesity, other therapies (preoperative chemotherapy or steroids) and HIV/AIDS. We also calculated a modified Charlson comorbidity index (CCI) as a marker of comorbidity [26].

\section{Hospital characteristics}

In France, establishments that perform thoracic procedures are classified as nonuniversity public hospitals, private hospitals and university hospitals. The explanatory variables of interest were hospital volume of 
pulmonary resections for lung cancer. For each hospital, the number of times each type of pulmonary resection was performed was calculated in the period from January 1, 2005 to December 31, 2013. Hospital volume was defined as the mean number of procedures performed per year, and was classified into three evenly sized groups (low: <13; medium: 13-43; and high: $>43$ ), following the calculation of quintiles and taking into account the literature.

We defined three periods: 2005-2007 (before Plan Cancer), 2008-2010 (implementation of new measures of Plan Cancer) and 2011-2013 (after Plan Cancer). The period could thus have had an impact on the quality of care [7].

\section{Outcome measurements}

IHM was defined as any patient who died in hospital (including transferred patients) within the first 30 days after the operation and those who died later during the same hospitalisation.

\section{Statistical analyses}

Descriptive data were expressed as $\mathrm{n}(\%)$ for qualitative variables and as mean $\pm \mathrm{sD}$ for continuous variables. Means were compared using a parametric test (ANOVA). We used a logistic regression model to construct prediction models for poor outcomes [27]. All models were constructed using backward stepwise variable selection. Step-down variable selection using Akaike's information criterion was used as a stopping rule [27]. Interaction effects were sought for all variables included in the model. The discriminative ability of the model was expressed by the area under the receiver operating characteristic curve (AUC) [28]. The reliability of the model was assessed using the Hosmer-Lemeshow goodness-of-fit test [29].

Uncertainty was estimated using a fixed-effect logistic regression model with an offset predictor model and centre as a categorical variable [26]. The standard error of the estimated coefficient, representing the mean outcome $(\sigma 2)$, indicated the uncertainty within the hospital [28].

We fitted a random-effect logistic regression model (multilevel model) to estimate unexplained heterogeneity, indicated by $\tau 2$ or the between-centre variance [28]. The variance indicates beyond-chance differences between hospitals.

To estimate rankability, we used the following formula: $\rho=\tau^{2} /\left(\tau^{2}+\right.$ median $\left.\sigma^{2}\right)$ [28]. Rankability can be interpreted as the part of heterogeneity between hospitals that is due to unexplained differences, and the rest is due to natural variation or chance [28]. All analyses were performed using SAS software (version 9.2; SAS Inc., Cary, NC, USA). Chi-squared tests were performed for qualitative variables.

\section{Results}

\section{Patient characteristics}

From January 2005 to December 2013, 76235 patients were operated on for lung cancer in France. Patient characteristics are reported in table 1. In the IHM group, there was a significantly greater proportion of males, of older patients and patients with a past history of liver and kidney disease, neurological disease, metabolic disease and haematological disease. Moreover, there were significantly more patients with a history of malignancies or HIV/AIDS in the IHM group (table 1).

\section{Management}

IHM was 3\% for limited resection, 3\% for lobectomy, 5\% for bilobectomy and $8.2 \%$ for pneumonectomy (table 1). The mortality rate for extended resection and sleeve resection was $6 \%$ and $5.5 \%$, respectively (table 1). Most resections (92.1\%) were performed by thoracotomy.

\section{Hospital characteristics}

Global crude IHM was $3.9 \%$ and decreased over the three periods from $4.3 \%$ to $3.5 \%(p<0.01)$, and the number of centres performing lung cancer surgery decreased from 296 to 209 centres $(\mathrm{p}<0.01)$ (table 2). There were 2975 (3.9\%) deaths during the hospital stay or within 30 days. The IHM rate was significantly higher for nonteaching hospital (4.7\%) than for teaching hospital (3.9\%) and private centres (3.7\%) (table 2).

Most of the patients (75\%) were operated on in centres performing $>43$ procedures per year. $\sim 60 \%$ of the centres $(n=206)$ had a mean $<13$ pulmonary resections per year, but these centres performed only $5.5 \%$ $(n=4151)$ of the total number of procedures over the three periods (table 2). IHM was significantly higher in low-volume centres ( $<13$ procedures per year) than in medium-volume centres (13-43 procedures per year) and high-volume centres ( $>43$ procedures per year) at $5.25 \%, 4 \%$ and $3.75 \%$, respectively $(\mathrm{p}<0.01)$ (table 2). The mean number of procedures per hospital increased over the three periods, but very high disparity remained between volumes in the different centres (figure 1). 
TABLE 1 Baseline characteristics associated with patient survival and in-hospital mortality

\begin{tabular}{|c|c|c|c|c|}
\hline & Total population & Survivors & In-hospital mortality & p-value \\
\hline Subjects & 76235 & 73260 & 2975 & \\
\hline \multicolumn{5}{|l|}{ Demographics } \\
\hline \multicolumn{5}{|l|}{ Sex } \\
\hline Male & 55204 & 52617 (95.3) & $2587(4.7)$ & \multirow[t]{2}{*}{$<0.001$} \\
\hline Female & 21031 & 20643 (98.2) & $388(1.8)$ & \\
\hline Age years & & $62.9 \pm 10.4$ & $67.9 \pm 9.5$ & $<0.001$ \\
\hline \multicolumn{5}{|l|}{ History } \\
\hline Pulmonary disease & 25863 & 23828 (92.2) & 2035 (7.8) & $<0.01$ \\
\hline Heart disease & 13506 & 12340 (91.4) & $1166(8.6)$ & $<0.01$ \\
\hline Peripheral vascular disease & 7891 & 7229 (91.4) & $662(8.4)$ & $<0.01$ \\
\hline Liver disease & 727 & $557(76.6)$ & $170(23.4)$ & $<0.01$ \\
\hline Neurological disease & 3495 & $3151(90.2)$ & $344(9.8)$ & $<0.01$ \\
\hline Metabolic disease & 6770 & 6318 (93.3) & $452(6.7)$ & $<0.01$ \\
\hline Renal disease & 1520 & $1332(97.6)$ & $188(12.4)$ & $<0.01$ \\
\hline Hematological disease & 2225 & $2020(90.8)$ & 205 (9.2) & $<0.01$ \\
\hline History of malignant disease & 29513 & 28112 (95.2) & $1401(4.7)$ & $<0.01$ \\
\hline HIV/AIDS & 896 & $696(77.7)$ & $200(22.3)$ & $<0.01$ \\
\hline Other treatment & 11148 & $10467(94)$ & $681(6)$ & $<0.01$ \\
\hline \multicolumn{5}{|l|}{ Modified CCl score } \\
\hline 0 & 25994 & 25456 (98) & $538(2)$ & \multirow[t]{4}{*}{$<0.001$} \\
\hline 1 & 10354 & $9999(96.6)$ & $355(3.4)$ & \\
\hline 2 & 9439 & $9062(96)$ & $377(4)$ & \\
\hline$\geqslant 3$ & 30441 & $28736(94.4)$ & $1705(5.6)$ & \\
\hline \multicolumn{5}{|l|}{ Surgical management } \\
\hline \multicolumn{5}{|l|}{ Type of pulmonary resection } \\
\hline Limited resection & 10144 & 9830 (97) & 314 (3) & \multirow[t]{4}{*}{$<0.001$} \\
\hline Lobectomy & 52254 & $50620(97)$ & $1634(3)$ & \\
\hline Bilobectomy & 3803 & $3596(95)$ & $207(5)$ & \\
\hline Pneumonectomy & 10034 & $9214(91.8)$ & $820(8.2)$ & \\
\hline \multicolumn{5}{|l|}{ Surgical approach } \\
\hline Thoracotomy & 70256 & $67425(96)$ & $2831(4)$ & $<0.01$ \\
\hline Extended resection & 12390 & $11653(94)$ & $737(6)$ & $<0.01$ \\
\hline Sleeve lobectomy & 2317 & 2189 (94.5) & $128(5.5)$ & $<0.01$ \\
\hline
\end{tabular}

Data are presented as $\mathrm{n}, \mathrm{n}(\%)$ or mean $\pm \mathrm{SD}$, unless otherwise stated. $\mathrm{CCl}$ : Charlson comorbidity index.

The variation in clinical characteristics and prognostic factors by hospital are reported in table 3 . The percentage of major resections, such as bilobectomy and pneumonectomy, varied substantially between hospitals $(0-6 \%$ and $0-16 \%$, respectively) (table 3$)$. Postoperative mortality varied substantially between hospitals (0-5\%) (table 3).

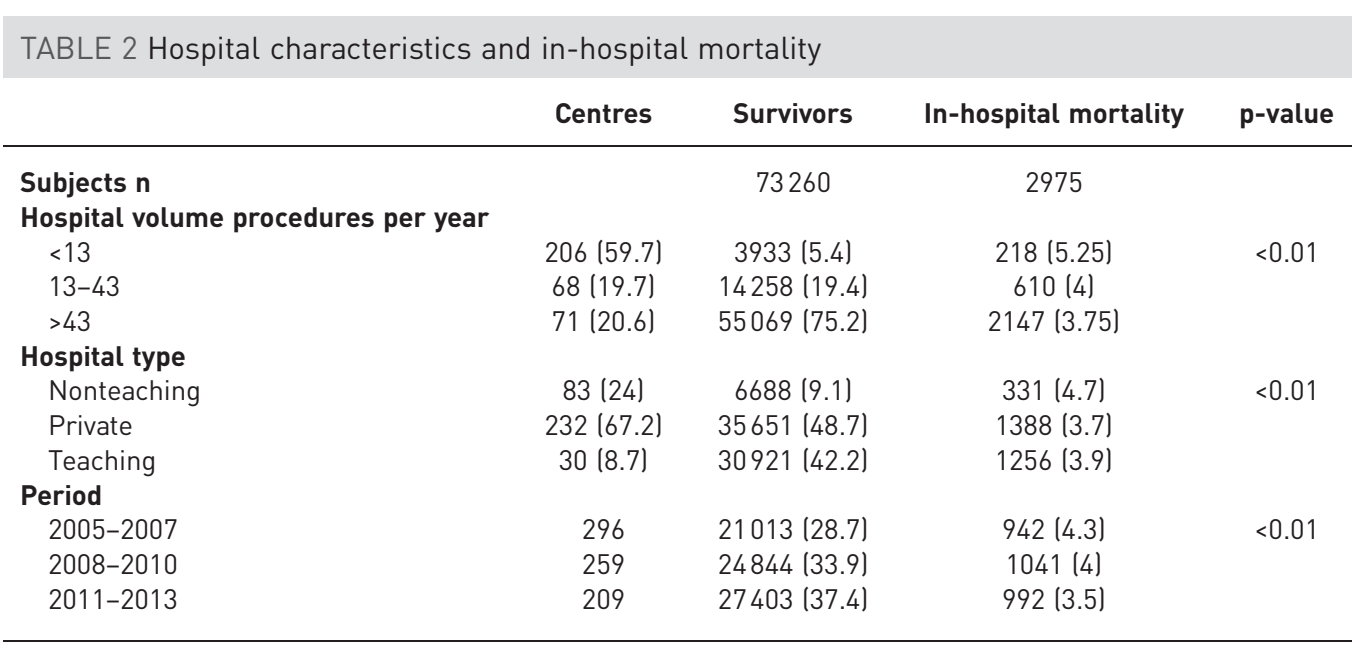

Data are presented as $n, n(\%)$ or mean \pm SD, unless otherwise stated. Total population $n=76235$. 
FIGURE 1 Distribution of number of procedures per year over the three periods: 2005-2007 (before Plan Cancer), 2008-2010 (implementation of new measures of Plan Cancer) and 2011-2013 (after Plan Cancer).

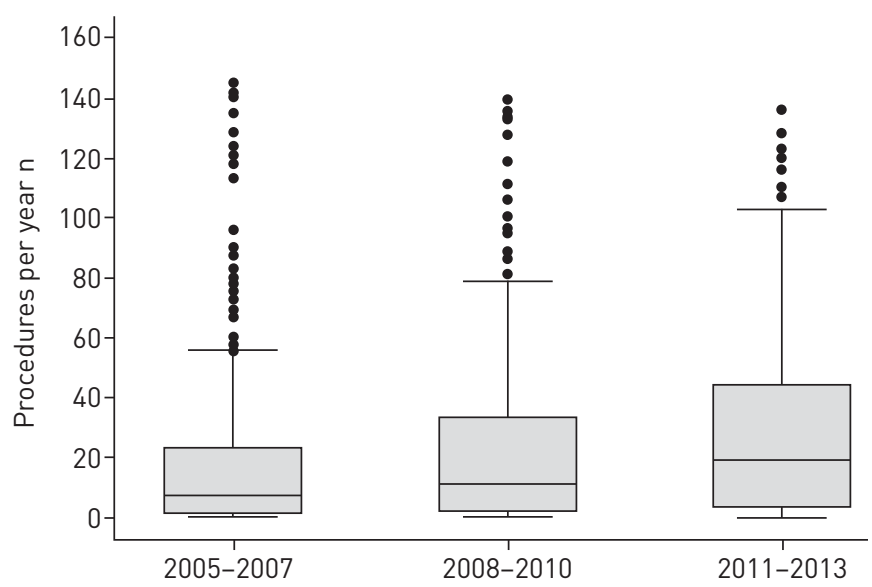

\section{Prognostic factors}

The results were the same in the fixed and random logistic models. The strongest predictors of poor outcomes were age, male sex, type of pulmonary resection, modified CCI score and comorbidities. The AUC of the model was 0.794 . The Hosmer-Lemeshow goodness-of-fit was nonsignificant for this model (Chi-squared 15.3, degrees of freedom 10; $\mathrm{p}=0.06$ ).

In the random logistic model, IHM was significantly linked to the volume of pulmonary resection: the adjusted (a)OR (95\% CI) was $1.5(1.2-1.8)$ for $<13$ procedures per year, 1.1 (0.94-1.3) for 13-43 procedures per year and 1 for $>43$ procedures per year (table 4 ). IHM was also significantly linked to the period of pulmonary resection: aOR (95\% CI) $1.08(0.98-1.2)$ for the period 2005-2007, 1 for the period 2008-2010 and $0.84(0.76-0.93)$ for the period 2011-2013 (table 4). IHM was not linked to the type of hospital (table 4).

The rankability was $63 \%$. This means that of the total variation between hospitals, $63 \%$ was not due to chance.

\section{Discussion}

In 2009, a new national strategy for quality improvement was implemented in French hospitals in order to improve the quality of the management of lung cancer patients. These measures concerned research, screening and the management of lung cancer patients. They also included for the first time the notion of volume of activity, to study its impact on markers of quality of care, such as IHM. This was based on numerous studies that have demonstrated a relationship between the number of surgical operations performed and the surgical outcomes for several surgical procedures including lung cancer resection [9, 10, 16, 18, 30-32].

\section{Patient characteristics and surgical management}

As previously described by many authors, our study highlighted that age, male sex and a high CCI score are prognostic factors of poor outcomes after lung cancer resection [13, 14, 33]. Moreover, IHM was significantly higher in bilobectomy and pneumonectomy because of the gravity of the resection itself [3].

\section{Hospital volume of activity}

We clearly demonstrated that IHM was higher in low-volume hospitals, where patients have a 1.5 higher risk of death during the postoperative period than patients undergoing surgery in high-volume centres,

TABLE 3 Variation in clinical characteristics and postoperative mortality by hospital ranked by the number of procedures performed per year

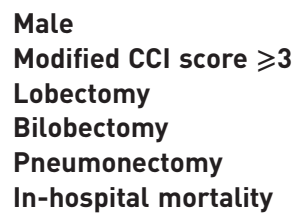

Data are presented as median (interquartile range). CCI: Charlson comorbidity index. 
TABLE 4 Random-effects logistic regression model

\begin{tabular}{|c|c|}
\hline \multicolumn{2}{|l|}{ Demographics } \\
\hline Age & $1.05(1.05-1.06)$ \\
\hline \multicolumn{2}{|l|}{ Sex } \\
\hline Female & 1 \\
\hline Male & $1.8(1.6-2)$ \\
\hline Modified $\mathrm{CCl}$ score 0 & 1 \\
\hline 1 & $0.88(0.76-1.02)$ \\
\hline 2 & $1.04(0.9-1.2)$ \\
\hline$\geqslant 3$ & $1.2(1.04-1.36)$ \\
\hline \multicolumn{2}{|l|}{ Past history } \\
\hline Pulmonary disease & $4(3.7-4.4)$ \\
\hline Neurological disease & $2(1.8-2.3)$ \\
\hline Liver disease & $7(5.7-8.5)$ \\
\hline Kidney disease & $2(1.75-2.5)$ \\
\hline Metabolic disease & $0.86(0.76-0.97)$ \\
\hline Haematological disease & $1.84(1.56-2.2)$ \\
\hline Other treatment & $1.18(1.07-1.3)$ \\
\hline \multicolumn{2}{|l|}{ Type of pulmonary resection } \\
\hline Limited resection & 1 \\
\hline Lobectomy & $1.05(0.92-1.2)$ \\
\hline Bilobectomy & $1.77(1.46-2.14)$ \\
\hline Pneumonectomy & $3(2.62-3.5)$ \\
\hline \multicolumn{2}{|l|}{ Centre characteristics } \\
\hline \multicolumn{2}{|c|}{ Hospital volume procedures per year } \\
\hline$<13$ & $1.5(1.2-1.8)$ \\
\hline $13-43$ & $1.1(0.94-1.3)$ \\
\hline$>43$ & 1 \\
\hline \multicolumn{2}{|l|}{ Hospital type } \\
\hline Nonteaching & 1 \\
\hline Private & $0.94(0.76-1.5)$ \\
\hline Teaching & $0.94(0.7-1.2)$ \\
\hline \multicolumn{2}{|l|}{ Period } \\
\hline $2005-2007$ & $1.08(0.98-1.2)$ \\
\hline $2008-2010$ & 1 \\
\hline $2011-2013$ & $0.84(0.76-0.93)$ \\
\hline
\end{tabular}

Data are presented as odds ratio $(95 \%$ confidence interval).

after adjustment for patient characteristics (including CCI score), the type of pulmonary resection, the period and hospital type. In 2012, von MEYENFELDT et al. [34] published a meta-analysis of 11 studies and highlighted significantly lower IHM in high-volume centres (OR 0.71, 95\% CI 0.62-0.81). In a few studies, the number of lung cancer resections was not significantly linked to IHM [10, 11, 17, 19]. This could be explained by small numbers of patients recorded in databases that are not representative of all lung cancer resections performed in a country, and by the thresholds used to define low- and high-volume centres. Indeed, in all the studies dealing with hospital volume for lung cancer surgery, the thresholds used to define low volume were quite heterogeneous. In our study, the threshold was $<13$ procedures a year, which is a very low level of activity to perform major lung cancer resections such as pneumonectomy and lobectomy. In most previous studies, low volume was defined as 9-34 pulmonary resections a year, but the threshold remains very difficult to establish for lung cancer surgery [9, 10, 16, 18, 19]. Moreover, a too-small number of patients could be insufficient to highlight a relationship between major surgery and IHM. In 2014, ReAmES et al. [35] reported IHM in 3282127 patients who underwent one of eight gastrointestinal, cardiac or vascular procedures, and brought to light a relationship between IHM and volume of activity in the different centres for all of the procedures studied.

In our study, almost $60 \%$ of the centres had low activity, but managed only $5.5 \%$ of the patients. When comparing the three periods of the study, we found that there was a decrease in the number of low-volume centres, thus highlighting the effect of the cancer plan. This trend has also been seen in Canada. Indeed, FInLEY et al. [19] studied IHM after lobectomy for lung cancer using data from the Canadian Institute for Health Information Discharge Database, and highlighted a similar trend. This trend could be explained on the one hand by the decrease in the use of major resection such as bilobectomy and pneumonectomy as described previously $[2,3]$, and by the improvement in the quality of care in cancer centres and potentially by the regionalisation of lung cancer surgery in Canada on the other hand $[19,36]$. 
In France, through the Plan Cancer, the French health institute defined the minimum volume for lung cancer surgery at 30 resections per year. In this study, we can see that there are still centres performing lung cancer surgery even though they have insufficient volumes of activity, which results in higher IHM.

The notion that IHM can be decreased by performing major cancer surgery in high-volume centres was reported 20 years ago and underlies the regionalisation policy of the French health institute, whose main objective is to improve the quality of care in major cancer resection [37]. However, FinLEY et al. reported no decrease in IHM when volume was increased within a given hospital, but a decrease in IHM in all centres of Ontario after the establishment of the regionalisation of lung cancer surgery in this province of Canada in 2004 [19].

The use of rankability was introduced by VAN HouwELINGEN et al. [28], for postoperative mortality in colorectal surgery. Rankability indicates which part of variation between hospitals is due to true differences and which part is due to chance [28]. The problem with ranking on crude hospital performance occurs when a rare event, such as IHM, is chosen [38]. Moreover, when some hospitals have small sample sizes, it makes the performance statistics unstable and the rank order unlikely to replicate [38]. In our study, rankability was $63 \%$, suggesting a huge difference in quality of care between hospitals and implying that $37 \%$ of the variation in IHM between hospitals was due to chance. At present in France, despite the Plan Cancer, there are still too many centres performing lung cancer resections even though they have low volumes of activity. We should logically consider reducing the number of centres managing lung cancer surgery in order to improve the quality of care, while respecting the needs of the French population.

Hospital type

We also found significantly higher IHM in nonteaching centres than in teaching hospitals and private centres $(4.7 \%, 3.9 \%$ and $3.7 \%$, respectively), but the type of hospital was no longer a significant prognostic factor for IHM after adjustment for the other factors (table 4). ROMANO and MARK [16] studied the influence of the level of teaching on IHM for lung cancer surgery, and found no difference between high-volume, low-volume and nonteaching centres. However, more recently, BHAMidipaTi et al. [12] studied outcomes after lung resection for 498099 patients according to the type of hospital in the United States. They found that mortality in thoracic residency hospitals was significantly lower than that in general surgery residency hospitals, thus suggesting that specialist thoracic residency was an independent prognostic indicator [12]. In our study, this tendency was not observed. Nevertheless, in French teaching hospitals, only certified thoracic surgeons perform lung cancer resection, which is not the case in nonteaching or private hospitals where the proportion of certified thoracic surgeons is lower, and more general thoracic surgeons practice lung cancer resection.

\section{Limitations}

There are limitations to our study. Given the reliance on ICD-10 codes for the selection of patients and the ascertainment of outcomes, there was a potential for misclassification- or underdetection-related bias, especially for comorbidities. Coding practices vary significantly among institutions. Nevertheless, coding quality is checked by medical information professionals in each hospital to correct diagnoses and increase the level of recorded comorbidity. No details were available concerning the accommodation facilities of the centre (number of beds in surgical departments and intensive care units, number of days spent in intensive care, number of nurses and medical practitioners), the organisation (tumour board meeting, application of recommendations, qualification of the surgeons and surgery performed by residents) and details about the management (type of thoracotomy and chemotherapy).

However, the demographic characteristics, risk factors and outcomes of the present study population were very similar to those in previous French studies from the Epithor database [3].

Unlike the Epithor database, the French Administrative Database does not record lung cancer information, such as the stage of the disease, preoperative forced expiratory volume or the American Society of Anesthesiologists score.

Despite these limitations, the strength of our results is related to the large size of our sample (73265 patients), with national recruitment. Finally, the results of the present study may be specific to the French healthcare system and cannot be generalised a priori to other countries with different healthcare systems.

\section{Conclusion}

IHM is significantly linked to hospital volume for lung cancer surgery in France, but not to the type of hospital. A significant decrease in IHM was observed in the period following implementation of the French initiative for clinical quality improvement. This was probably linked to the decrease in the number of centres allowed to perform lung cancer surgery (authorisation delivered according to the volume of activity), 
the increase in the volume of activity for authorised centres and better preoperative selection of patients. However, many centres are still performing $<13$ pulmonary resections a year for lung cancer.

\section{Acknowledgements}

The authors would like to thank Philip Bastable (Dijon University Hospital, Dijon, France) for revising the manuscript.

\section{References}

1 Howington JA, Blum MG, Chang AC, et al. Treatment of stage I and II non-small cell lung cancer: diagnosis and management of lung cancer, 3rd ed: American College of Chest Physicians evidence-based clinical practice guidelines. Chest 2013; 143: Suppl. 5, e278S-e313S.

2 Strand T-E, Bartnes K, Rostad H. National trends in lung cancer surgery. Eur J Cardiothorac Surg 2012; 42: 355-358.

3 Morgant M-C, Pagès P-B, Orsini B, et al. Time trends in surgery for lung cancer in France from 2005 to 2012: a nationwide study. Eur Respir J 2015; 46: 1131-1139.

4 Donabedian A. The quality of care. How can it be assessed? JAMA 1988; 260: 1743-1748.

5 Mant J. Process versus outcome indicators in the assessment of quality of health care. Int J Qual Health Care 2001; 13: 475-480.

6 Iezzoni LJ, ed. Risk Adjustment for Measuring Healthcare Outcomes. 3rd Edn. Chicago, Health Administration Press, 2003.

7 Ministère de l'Enseignement Supérieur et de la Recherche, Ministère de la Santé et des Sports. Plan Cancer 2009-2013. www.e-cancer.fr/Expertises-et-publications/Catalogue-des-publications/Plan-cancer-2009-2013 Date last accessed: March 1, 2016. Date last updated: September 30, 2012.

8 Ministère de la Santé et des Solidarités. Arrêté du 29 mars 2007 fixant les seuils d'activité minimale annuelle applicables à l'activité de soins de traitement du cancer [Decree of 29 March 2007 laying down annual minimum activity thresholds applicable to the activity of cancer treatment care]. Journal Officiel de la République Française. www.legifrance.gouv.fr/jopdf/common/jo_pdf.jsp?numJO=0\&dateJO=20070330\&numTexte=68\&pageDebut $=05963$ \&pageFin=05964 Date last accessed: March 1, 2016. Date last updated: March 30, 2007.

9 Birkmeyer JD, Siewers AE, Finlayson EVA, et al. Hospital volume and surgical mortality in the United States. N Engl J Med 2002; 346: 1128-1137.

10 Finlayson EVA, Goodney PP, Birkmeyer JD. Hospital volume and operative mortality in cancer surgery: a national study. Arch Surg 2003; 138: 721-725.

11 Lien Y-C, Huang M-T, Lin H-C. Association between surgeon and hospital volume and in-hospital fatalities after lung cancer resections: the experience of an Asian country. Ann Thorac Surg 2007; 83: 1837-1843.

12 Bhamidipati CM, Stukenborg GJ, Ailawadi G, et al. Pulmonary resections performed at hospitals with thoracic surgery residency programs have superior outcomes. J Thorac Cardiovasc Surg 2013; 145: 60-66.

13 Rosen JE, Hancock JG, Kim AW, et al. Predictors of mortality after surgical management of lung cancer in the National Cancer Database. Ann Thorac Surg 2014; 98: 1953-1960.

14 Green A, Hauge J, Iachina M, et al. The mortality after surgery in primary lung cancer: results from the Danish Lung Cancer Registry. Eur J Cardiothorac Surg 2016; 49: 589-594.

15 Dillman RO, McClure SE. Steadily improving survival in lung cancer. Clin Lung Cancer 2014; 15: 331-337.

16 Romano PS, Mark DH. Patient and hospital characteristics related to in-hospital mortality after lung cancer resection. Chest 1992; 101: 1332-1337.

17 Freixinet JL, Julià-Serdà G, Rodríguez PM, et al. Hospital volume: operative morbidity, mortality and survival in thoracotomy for lung cancer. A Spanish multicenter study of 2994 cases. Eur J Cardiothorac Surg 2006; 29: 20-25.

18 Otake $\mathrm{H}$, Yasunaga $\mathrm{H}$, Horiguchi $\mathrm{H}$, et al. Impact of hospital volume on chest tube duration, length of stay, and mortality after lobectomy. Ann Thorac Surg 2011; 92: 1069-1074.

19 Finley CJ, Bendzsak A, Tomlinson G, et al. The effect of regionalization on outcome in pulmonary lobectomy: a Canadian national study. J Thorac Cardiovasc Surg 2010; 140: 757-763.

20 Lorgis L, Cottenet J, Molins G, et al. Outcomes after acute myocardial infarction in HIV-infected patients: analysis of data from a French nationwide hospital medical information database. Circulation 2013; 127: 1767-1774.

21 Lainay C, Benzenine E, Durier J, et al. Hospitalization within the first year after stroke: the Dijon stroke registry. Stroke 2015; 46: 190-196.

22 Abdulmalak C, Cottenet J, Beltramo G, et al. Haemoptysis in adults: a 5-year study using the French nationwide hospital administrative database. Eur Respir J 2015; 46: 503-511.

23 Iezzoni LI. Assessing quality using administrative data. Ann Intern Med 1997; 127: 666-674.

24 World Health Organization. International Statistical Classification of Diseases and Related Health Problems 10th Revision. http://apps.who.int/classifications/icd10/browse/2016/en Date last accessed: March 1, 2016. Last updated: 2016.

25 Travis WD, Brambilla E, Müller-Hermelink HK, et al. Pathology and Genetics: Tumours of the Lung, Pleura, Thymus and Heart. Lyon, IARC Press, 2004.

26 Charlson M, Szatrowski TP, Peterson J, et al. Validation of a combined comorbidity index. J Clin Epidemiol 1994; 47: 1245-1251.

27 Steyerberg EW. Clinical Prediction Models. A Practical Approach to Development, Validating and Updating. New York, Springer-Verlag, 2009.

28 Van Houwelingen HC, Brand R, Louis TA. Empirical Bayes methods for monitoring health care quality. www.lumc. nl/sub/3020/att/empiricalbayes.pdf Date last accessed: December 20, 2012.

29 Hosmer DW, Lemeshow S. A goodness-of-fit test for multiple regression model. Commun Stat 1980; A10: 1043-1069.

30 Killeen SD, O'Sullivan MJ, Coffey JC, et al. Provider volume and outcomes for oncological procedures. Br J Surg 2005; 92: 389-402.

31 Hannan EL, Radzyner M, Rubin D, et al. The influence of hospital and surgeon volume on in-hospital mortality for colectomy, gastrectomy, and lung lobectomy in patients with cancer. Surgery 2002; 131: 6-15.

32 Bach PB, Cramer LD, Schrag D, et al. The influence of hospital volume on survival after resection for lung cancer. N Engl J Med 2001; 345: 181-188. 
33 Birim O, Kappetein AP, Bogers AJJC. Charlson comorbidity index as a predictor of long-term outcome after surgery for nonsmall cell lung cancer. Eur J Cardiothorac Surg 2005; 28: 759-762.

34 von Meyenfeldt EM, Gooiker GA, van Gijn W, et al. The relationship between volume or surgeon specialty and outcome in the surgical treatment of lung cancer: a systematic review and meta-analysis. J Thorac Oncol 2012; 7 : $1170-1178$.

35 Reames BN, Ghaferi AA, Birkmeyer JD, et al. Hospital volume and operative mortality in the modern era. Ann Surg 2014; 260: 244-251.

36 Birkmeyer NJO, Goodney PP, Stukel TA, et al. Do cancer centers designated by the National Cancer Institute have better surgical outcomes? Cancer 2005; 103: 435-441.

37 Birkmeyer JD. Should we regionalize major surgery? Potential benefits and policy considerations. J Am Coll Surg 2000; 190: 341-349.

38 van Dishoeck A-M, Lingsma HF, Mackenbach JP, et al. Random variation and rankability of hospitals using outcome indicators. BMJ Qual Saf 2011; 20: 869-874. 